\title{
In-office bleaching effects on the pulp flow and tooth sensitivity - case series
}

\section{Andrés Felipe CARTAGENA(a) Sibelli Olivieri PARREIRAS(a) Alessandro Dourado LOGUERCIO(b) Alessandra REIS(b) Nara Hellen CAMPANHA(c)}

(a) Universidade Estadual de Ponta Grossa UEPG, School of Dentistry, Posgraduate Program in Dentistry, Ponta Grossa, PR, Brazil.

(b) Universidade Estadual de Ponta Grossa - UEPG, School of Dentistry, Department of Operative Dentistry, Ponta Grossa, PR, Brazil.

(c) Universidade Estadual de Ponta Grossa UEPG, School of Dentistry, Department of Prosthodontics, Ponta Grossa, PR, Brazil.

Declaration of Interests: The authors certify that they have no commercial or associative interest that represents a conflict of interest in connection with the manuscript.

Corresponding Author:

Alessandra Reis

E-mail: reisale@hotmail.com

DOI: 10.1590/1807-3107BOR-2015.vol29.0026

Submitted: May 13, 2014

Accepted for publication: Aug 29, 2014

Last revision: Dec 08, 2014
Abstract: Laser Doppler flowmetry (LDF) is a noninvasive method capable of evaluating variations in pulp blood flow (PBF) and pulp vitality. This method has thus far not been used to assess changes in blood flow after in-office bleaching. The aim of this case series report was to measure changes in PBF by LDF in the upper central incisor of three patients submitted to in-office bleaching. The buccal surfaces of the upper arch were bleached with a single session of $35 \%$ hydrogen peroxide gel with three 15 -min applications. The color was recorded using a value-oriented Vita shade guide before in-office bleaching and one week after the procedure. The tooth sensitivity (TS) in a verbal scale was reported, and PBF was assessed by LDF before, immediately, and one week after the bleaching session. The lower arch was submitted to dental bleaching but not used for data assessment. A whitening degree of 3 to 4 shade guide units was detected. All participants experienced moderate to considerable TS after the procedure. The PBF readings reduced $20 \%$ to $40 \%$ immediately after bleaching. One week postbleaching, TS and PBF were shown to be equal to baseline values. A reversible decrease of PBF was detected immediately after bleaching, which recovered to the baseline values or showed a slight increase sooner than one week post-bleaching. The LDF method allows detection of pulp blood changes in teeth submitted to in-office bleaching, but further studies are still required.

Keywords: Laser-Doppler Flowmetry; Dentin Sensitivity; Tooth Bleaching.

\section{Introduction}

Dental bleaching is a widely used cosmetic protocol employed in daily clinical practice. This technique fulfills the patient's expectations in the search for a whiter smile. Currently, there are two main dentistsupervised techniques: the at-home or in-office bleaching. The at-home bleaching is performed using a custom-fitted bleaching tray filled with a low concentration of hydrogen peroxide (HP) or carbamide peroxide. The patients are instructed to wear the bleaching tray daily, for periods ranging from 1 to $8 \mathrm{~h}$ from 2 to 6 weeks. ${ }^{1}$

In spite of the safety and effectiveness of this technique to whiten teeth, some patients do not want to use the trays even for shorter periods of time. In this clinical scenario, in-office bleaching using higher concentrations 
of $\mathrm{HP}$ is the alternative protocol. This protocol has been shown to produce color changes more rapidly. ${ }^{2}$

Fortunately, as long as the in-office bleaching is performed more than once, bleaching of approximately 5 to 8 shade guide units is possible. ${ }^{2,3,4}$ Stable results were also demonstrated after 9 and 24 months., ${ }^{4,5}$ Even though professional-assisted dental bleaching is considered a safe procedure, tooth sensitivity (TS) is a remarkably common side effect reported by patients. ${ }^{13,4}$ More than $70 \%$ of patients who undergo in-office bleaching complain of TS, ${ }^{6}$ which leads some patients to forego treatment. ${ }^{7}$

The uncomfortable and painful bleaching-induced TS is likely the result of pulp insult by the rapid diffusion of HP molecules. Pulp damage can trigger an inflammatory reaction that leads to the release of cell-derived factors such as adenosine triphosphate ${ }^{8}$ neuropeptides, and prostaglandins, which excite or sensitize pulpal nociceptors., ${ }^{9,11}$ This inflammatory reaction also induces vasodilatation and increased pulpal blood flow (PBF). 12,13,14

In a recent histological study, Costa et al. ${ }^{15}$ evaluated the effects of HP on pulp cells and showed notable damage to the pulp tissue, with mild inflammatory changes as well as sites of pulp necrosis in lower incisors. This study has raised concerns about pulp vitality of teeth soon after in-office bleaching. Although there are many clinical studies on tooth bleaching, the pulp vitality of bleached teeth has not been assessed thus far. This may be due to the fact that most of the current methods employed to assess pulp vitality may give a high percentage of false-positive results. ${ }^{16,17}$

An interesting approach is noninvasive laser Doppler, which can monitor PBF changes through measuring the velocity of red blood cells in capillaries. Additionally, it is an objective, semiquantitative, and painless method. This method was first used on human teeth by Gazelius et al. ${ }^{18}$ in 1986. The value of this method for assessing pulp vitality has been well documented ${ }^{19}$ but its high costs and difficulty of use in clinical practice have delayed its wide-scale introduction. ${ }^{16,20}$ Therefore, the aim of this preliminary case series report was to assess the viability of using the laser Doppler to monitor PBF in teeth submitted to in-office bleaching.

\section{Methodology}

The Scientific Review Committee and the Committee for the Protection of Human Beings at the local university approved this clinical investigation (protocol no. 172.988).

Three patients from 18 to 24 years old sought clinical assistance at the School of Dentistry of the Universidade Estadual de Ponta Grossa - UEPG (Paraná, Brazil). All patients were not satisfied with the yellowing color of their teeth. The participants should have caries-free maxillary teeth without restorations. Participants who had previously undergone tooth-whitening procedures, presenting internal tooth discoloration, taking any medicine, or presenting any pulpal pathology or participants whose teeth had enamel cracks were not included in the study. After anamnesis and a clinical evaluation, the patients decided to have in-office bleaching performed because they did not complain about using the trays. All participants were informed via written consent about possible sensitivity and other conditions or likely side effects such as gingival burning.

Two weeks before the bleaching procedures, all patients received a dental screening and prophylaxis with pumice and water in a rubber cup. During this visit, possible TS due to cold air was assessed using air spray on all teeth to be bleached for $3 \mathrm{~s}$. None of the patients complained about TS or reported a history of trauma or endodontic treatment on any tooth.

During the in-office bleaching session, the gingival tissue of the teeth from the upper arch was isolated with a light-curing resin dam (Top Dam, FGM, Joinville, Brazil, batch 260712). The 35\% HP gel (Whiteness HP Maxx, FGM, batch 260712) was manipulated according to the manufacturer's directions and applied on the buccal surfaces of all upper anterior teeth and premolars. The in-office bleaching agent was refreshed every 15 min during the 45-min application period. The lower arch was also submitted to bleaching one week after the end of the upper arch treatment but was not used for data assessment.

The color was recorded at baseline and 1 week after the bleaching sessions using a value-oriented Vita shade guide (VITA Classical Shade Guide, Vita Zahnfabrik, Bad Säckingen, Germany). The 
shade evaluation was done in a single room with artificial lighting by a single calibrated operator. For this examination, the shade guide's 16 tabs were arranged from the highest (B1) to lowest (C4) value, making the color A3, number 9. This allowed for the calculation of the variation of the shade guide units ( $\triangle S G U)$. The measurement area of interest for shade matching was the middle onethird of the buccal surface of the central upper left incisor (tooth 21). The patients were instructed to record their perception of TS on a 5-point verbal rating scale during bleaching up to 1 week after the bleaching session. They kept a daily record of whether they experienced sensitivity using the following criteria: $0=$ none, $1=$ mild, $2=$ moderate, $3=$ considerable, and $4=$ severe .

The PBF was recorded at baseline, immediately, and 1 week after the bleaching session in the central upper left incisor with LDF (MoorLAB/FloLAB; Moor Instruments Ltd., Axminster, England). ${ }^{20}$ The device was calibrated against a Brownian motion medium and zeroed against a static reflector. The laser source was $780 \mathrm{~nm}$, with $0.5 \mathrm{~mm}$ of fiber separation in the MoorLAB probe P13, with $3.1 \mathrm{kHz}$ as the primary bandwidth for a filter set to a 0.1-s time output constant.

The patients rested in a supine position for 3-5 minutes before starting the LDF test. To ensure accurate and reproducible positioning of the probe at each examination, a matrix of high-viscosity silicone was prepared for each patient (Zetalabor, Zhermack, Polesine Badia, Italy). This silicone matrix was fashioned with appropriate holes with the corresponding diameter of the flowmeter probe tip (Figure 1). The probe was positioned so that it was perpendicular to the enamel surface, with its center $2 \mathrm{~mm}$ from the gingival margin and over the central long axis of the crown of the tooth.

The flux signal from the blood flow monitor was transferred to a computer, analyzed using the Moorsoft program (Moor Instruments, Axminster, England), and transformed in arbitrary perfusion units. Recordings of PBF were made for $90 \mathrm{~s}$ at each period. The perfusion units measured at the different periods were relative to the perfusion unit at baseline, considered to be $100 \%$.

\section{Results}

A whitening of approximately 3 to 4 SGU was detected for the three patients after the in-office bleaching (Table 1). Some changes were found in red blood flow and TS after the in-office bleaching. The incidence of TS ranged from moderate to considerable (Table 1) after bleaching. One week after the procedure, the patients reported only mild TS. A $20 \%$ to $40 \%$ reduction of the $\mathrm{PBF}$ was observed immediately after in-office bleaching (Figure 2). One week post-bleaching, the values of PBF either reached the baseline values or showed a slight increase.

\section{Discussion}

$\mathrm{HP}$ is the active molecule that causes tooth bleaching. This study demonstrated a significant improvement in shade compared with the baseline color. Previous studies have demonstrated a higher whitening degree ${ }^{2,3}$ than the current one. This lower whitening effect is probably due to the fact that only one bleaching session was performed in this study.

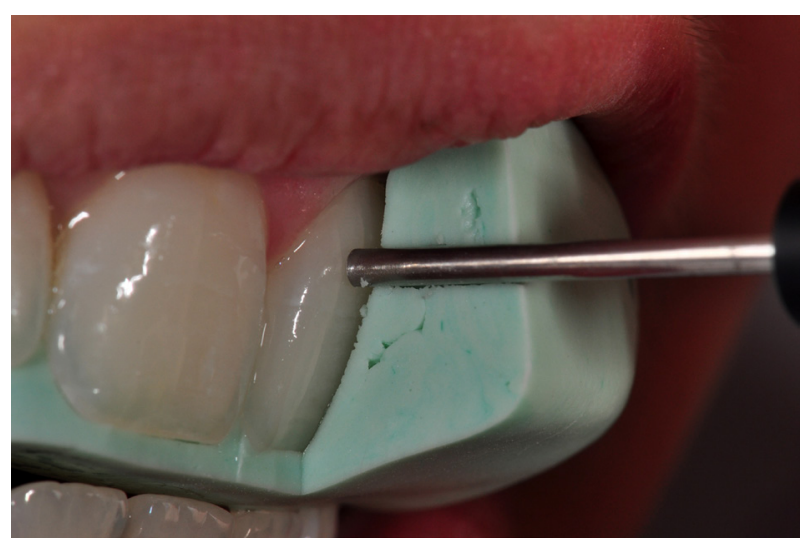

Figure 1. Transversal view of the probe tip positioned in the silicone matrix.

Table 1. TS scores and SGU at different assessment points for each participant.

\begin{tabular}{lcccccc}
\hline & \multicolumn{5}{c}{ Patients } \\
\cline { 2 - 7 } Time Assessment & \multicolumn{5}{c}{ TS } & \multicolumn{5}{c}{$\Delta$ SGU } \\
\cline { 2 - 7 } & P1 & P2 & P3 & P1 & P2 & P3 \\
\hline Baseline & 0 & 0 & 0 & 5 & 6 & 5 \\
After bleaching & 2 & 3 & 3 & - & - & - \\
1 week after bleaching & 1 & 0 & 1 & 2 & 2 & 1 \\
\hline
\end{tabular}




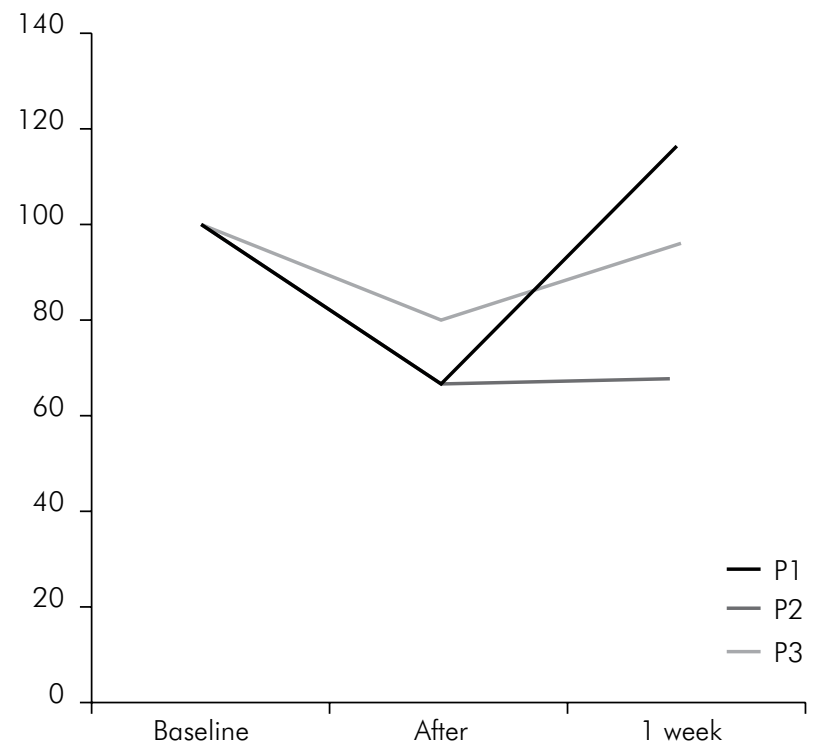

Figure 2. Relative percentage of pulp blood flow in the upper left central incisor of each participant.

Unfortunately, HP action is not limited to dental hard tissues. HP can also reach the pulp chamber, where it was shown to reduce cell proliferation, metabolism, and viability. ${ }^{21}$ Additionally, it may also reduce the pulp-reparative capacity ${ }^{22}$ and induce pulp inflammation, which may be responsible for the TS experienced by the patients during in-office bleaching. ${ }^{1}$

All three participants from this study experienced moderate to considerable TS that decreased to a mild level one week after bleaching. The literature usually reports that TS normally persists for up to four days after bleaching, ${ }^{2}$ but durations of up to 39 days have been reported. ${ }^{7}$ The inflammatory process produced by tooth bleaching usually lasts for approximately 2 weeks. During this time, proinflammatory cytokine release continues, which, in some cases, could perpetuate substance P release for longer periods of time and therefore cause postbleaching symptoms in some cases. ${ }^{10,11}$

The optical method herein used enables the recording of the number and velocity of particles conveyed by a fluid flow. ${ }^{16}$ As red blood cells represent the vast majority of moving cells within the dental structure tooth, measurement of the Doppler-shifted, backscattered light serves as an index of PBF. This device is capable of evaluating the dynamic changes in blood flow in a small volume of tissue (about $\left.1 \mathrm{~mm}^{3}\right){ }^{23}$
As most studies on LDF have been undertaken on anterior teeth, including central and lateral incisors and also canines, ${ }^{16,19}$ this preliminary case report followed the literature trend. Additionally, we opted to measure it on the central incisor as most of clinical trials on bleaching employ this tooth as a reference for assessment of the whitening efficacy. Besides that, blood flow varies in different teeth from the same patient, ${ }^{19}$ therefore, when the aim is to compare different procedures, a single tooth should be selected for evaluation to avoid adding intertooth variability on blood flow response.

There is a great variability in PBF among patients, ${ }^{19}$ and this is the reason that the variation in the pulp flow in the present study was reported relative to the flowmeter at baseline for each patient in an intraindividual comparison. This reduces the variability of the measurement and is a viable method to assess the effect of some restorative and cosmetic protocols on pulp vitality, including bleaching protocols.

When pulp is directly injured, such as during pulp exposure, neuropeptide levels increase in the pulp and trigeminal ganglia, ${ }^{24}$ These neuropeptides may be released because of the stimulation from dental procedures, causing vasodilatation and increased $\mathrm{PBF}^{14,25}$ However, in this study, a decrease in the PBF in the patients was detected, which is in agreement with the findings of Chen and Abbott. ${ }^{17}$ Contrary to other conjunctive tissues, the inflammatory vascular reactions in the dental pulp take place in a rigid, enclosed dentin chamber. Any increase in blood flow and vascular permeability can cause major changes in the pressure inside the pulp chamber, which may compress blood vessels and lead to the decrease in the pulp flow ${ }^{14}$ detected by the laser Doppler device.

However, one week after tooth bleaching, the values either reached baseline values or remained slightly higher. This variability may be explained based on individual variation of the same injury. Perhaps the slight increase in PBF detected in one patient explains changes in the microcirculatory and micromorphology dynamics of the pulp tissue after the inflammatory process. ${ }^{26,27,28}$ Further studies with a larger sample size should be conducted to investigate whether the dynamics of pulp flow are affected by dental bleaching and other dental procedures. 
The difficulties of such a device should also be mentioned. Variables such as the calibration of the flowmeter, the position of the probe and patient, the patient's systemic pressure, the patient's use of drugs, and ambient temperature can all influence PBF, but they can be controlled clinically. However, there are other factors that are not under clinical control. For instance, the individual variations in the PBF and neurovascular response, the differences in the optical properties of the dental structure (fluorescence, opalescence, etc.), tooth morphology, and the surrounding tissues can cause variability in the measurements. ${ }^{16}$

Another limitation of the present study is that LDF was not compared to other methods. Unfortunately, there is no noninvasive gold standard in the literature for evaluation of pulp blood changes. Pulse oximetry and electric tests are other options to assess pulp vitality; however, previous studies suggest that LDF is more sensitive and specific in human teeth. ${ }^{29}$

The objective assessment of pain perception or sensitivity is limited due to its nature. There are many simple and direct methods to measure this perception of pain, as the VAS scale used in this study has done.

\section{References}

1. Basting R, Amaral F, Franca F, Florio F. Clinical comparative study of the effectiveness of and tooth sensitivity to $10 \%$ and $20 \%$ carbamide peroxide home-use and $35 \%$ and $38 \%$ hydrogen peroxide in-office bleaching materials containing desensitizing agents. Oper Dent. 2012 Sep-Oct;37(5):464-73.

2. Bernardon JK, Sartori N, Ballarin A, Perdigao J, Lopes GC, Baratieri LN. Clinical performance of vital bleaching techniques. Oper Dent. 2010 Jan-Feb;35(1):3-10.

3. Kossatz S, Dalanhol AP, Cunha T, Loguercio A, Reis A. Effect of light activation on tooth sensitivity after in-office bleaching. Oper Dent. 2011 May-Jun;36(3):251-7.

4. Tay LY, Kose C, Herrera DR, Reis A, Loguercio AD. Long-term efficacy of in-office and at-home bleaching: a 2-year double-blind randomized clinical trial. Am J Dent. 2012 Aug;25(4):199-204.

5. Giachetti L, Bertini F, Bambi C, Nieri M, Scaminaci Russo D. A randomized clinical trial comparing at-home and in-office tooth whitening techniques: a nine-month follow-up. J Am Dent Assoc. 2010 Nov;141(11):1357-64.

6. Reis A, Dalanhol AP, Cunha TS, Kossatz S, Loguercio AD. Assessment of tooth sensitivity using a desensitizer before light-activated bleaching. Oper Dent. 2011 Jan-Feb;36(1):12-7.
However, these methods are considered subjective, so accurate assessment of pain remains a challenge. ${ }^{30}$ With the results of this preliminary study, we observed a relationship between the changes in PBF and the perceived sensitivity reported by patients. Thus, despite the limitations of LDF, it could be used to increase the reliability of pain scales as instruments to assess the perception of TS.

Finally, future studies should be conducted to evaluate PBF between teeth of the same patient and between individuals as well as to correlate PBF with TS. The preliminary results of this study suggest that the use of LDF is a viable alternative to assess the changes in pulp tissue that may occur due to tooth bleaching in shorter periods; however, its current use requires further study.

\section{Conclusion}

LDF is a viable alternative to assess blood flow changes in teeth submitted to vital tooth bleaching; however, it requires further study using a larger sample size. Immediately after bleaching, reduced blood flow was observed, but this was reversed one week after of intervention.

7. Leonard RHJr, Haywood VB, Phillips C. Risk factors for developing tooth sensitivity and gingival irritation associated with nightguard vital bleaching. Quintessence Int. 1997 Aug;28(8):527-34.

8. Cook SP, McCleskey EW. Cell damage excites nociceptors through release of cytosolic ATP. Pain. 2002 Jan;95(1-2):41-7.

9. Markowitz K. Pretty painful: why does tooth bleaching hurt? Med Hypotheses. 2010 May;74(5):835-40.

10. Bowles WR, Withrow JC, Lepinski AM, Hargreaves KM. Tissue levels of immunoreactive substance $P$ are increased in patients with irreversible pulpitis. J Endod. 2003 Apr;29(4):265-7.

11. Patel T, Park SH, Lin LM, Chiappelli F. Huang GT. Substance $P$ induces interleukin-8 secretion from human dental pulp cells. Oral Surg Oral Med Oral Pathol Oral Radiol Endod. 2003 Oct;96(4):478-85.

12. Caviedes-Bucheli J, Ariza-Garcia G, Restrepo-Mendez S, Rios-Osorio N, Lombana N, Munoz HR. The effect of tooth bleaching on substance P expression in human dental pulp. J Endod. 2008 Dec;34(12):1462-5.

13. Caviedes-Bucheli J, Lombana N, Azuero-Holguin MM, Munoz HR. Quantification of neuropeptides (calcitonin gene-related peptide, substance $\mathrm{P}$, neurokinin $\mathrm{A}$, neuropeptide $\mathrm{Y}$ and vasoactive intestinal polypeptide) expressed in healthy and inflamed human dental pulp. Int Endod J. 2006 May;39(5):394-400. 
14. Kim S. Neurovascular interactions in the dental pulp in health and inflammation. J Endod. 1990 Feb;16(2):48-53.

15. Costa CA, Riehl H, Kina JF, Sacono NT, Hebling J. Human pulp responses to in-office tooth bleaching. Oral Surg Oral Med Oral Pathol Oral Radiol Endod. 2010 Apr;109(4):e59-64.

16. Jafarzadeh H. Laser Doppler flowmetry in endodontics: a review. Int Endod J. 2009 Jun;42(6):476-90.

17. Chen E, Abbott PV. Evaluation of accuracy, reliability, and repeatability of five dental pulp tests. J Endod. 2011 Dec;37(12):1619-23.

18. Gazelius B, Olgart L, Edwall B, Edwall L. Non-invasive recording of blood flow in human dental pulp. Endod Dent Traumatol. 1986 Oct;2(5):219-21.

19. Norer B, Kranewitter R, Emshoff R. Pulpal blood-flow characteristics of maxillary tooth morphotypes as assessed with laser Doppler flowmetry. Oral Surg Oral Med Oral Pathol Oral Radiol Endod. 1999 Jan;87(1):88-92.

20. Roy E, Alliot-Licht B, Dajean-Trutaud S, Fraysse C, Jean A, Armengol V. Evaluation of the ability of laser Doppler flowmetry for the assessment of pulp vitality in general dental practice. Oral Surg Oral Med Oral Pathol Oral Radiol Endod. 2008 Oct;106(4):615-20.

21. Min KS, Lee HJ, Kim SH, Lee SK, Kim HR, Pae HO, et al. Hydrogen peroxide induces heme oxygenase- 1 and dentin sialophosphoprotein mRNA in human pulp cells. J Endod. 2008 Aug;34(8):983-9.

22. Goldberg M, Smith AJ. Cells and extracellular matrices of dentin and pulp: a biological basis for repair and tissue engineering. Crit Rev Oral Biol Med. 2004 Jan;15(1):13-27.
23. Vongsavan N, Matthews B. Some aspects of the use of laser Doppler flow meters for recording tissue blood flow. Exp Physiol. 1993 Jan;78(1):1-14.

24. Buck S, Reese K, Hargreaves KM. Pulpal exposure alters neuropeptide levels in inflamed dental pulp and trigeminal ganglia: evaluation of axonal transport. J Endod. 1999 Nov;25(11):718-21.

25. Heyeraas KJ, Kim S, Raab WH, Byers MR, Liu M. Effect of electrical tooth stimulation on blood flow, interstitial fluid pressure and substance P and CGRP-immunoreactive nerve fibers in the low compliant cat dental pulp. Microvasc Res. 1994 May;47(3):329-43.

26. Matheny JL, Abrams H, Johnson DT, Roth GI. Microcirculatory dynamics in experimental human gingivitis. J Clin Periodontol. 1993 Sep;20(8):578-83.

27. Mesaros SV, Trope M. Revascularization of traumatized teeth assessed by laser Doppler flowmetry: a case report. Endod Dent Traumatol. 1997 Feb;13(1):24-30.

28. Núñez SC, Nogueira GE, Ribeiro MS, Garcez AS, LageMarques JL. He-Ne laser effects on blood microcirculation during wound healing: a method of in vivo study through laser Doppler flowmetry. Lasers Surg Med. 2004;35(5):363-8.

29. Karayilmaz H, Kirzioğlu Z. Comparison of the reliability of laser Doppler flowmetry, pulse oximetry and electric pulp tester in assessing the pulp vitality of human teeth. J Oral Rehabil. 2011 May;38(5):340-7.

30. Breivik H, Borchgrevink PC, Allen SM, Rosseland LA, Romundstad L, Hals EK, et al. Assessment of pain. Br J Anaesth. 2008 Jul;101(1):17-24. 